\title{
PROPRIEDADES DE RESISTÊNCIA E RIGIDEZ À FLEXÃO ESTÁTICA DE PAINÉIS OSB E COMPENSADOS ${ }^{1}$
}

\author{
Geraldo Bortoletto Júnior ${ }^{2}$, José Nivaldo Garcia²
}

\begin{abstract}
RESUMO - Em uma indústria de móveis estofados, localizada em Piracicaba, SP, foram coletados aleatoriamente corpos-de-prova de painéis compensados e OSB para determinação das propriedades de resistência (MOR) e rigidez (MOE) à flexão estática, com as finalidades de compará-las e avaliar a possibilidade de substituição direta do compensado, utilizado tradicionalmente nas partes estruturais dos móveis, por OSB. Os ensaios foram realizados com base nas prescrições da norma brasileira para compensados (NBR 9533), similar à norma americana ASTM D 1037. A análise dos resultados de MOR (paralelo e perpendicular) e MOE (perpendicular) revelou que os painéis de compensado foram significativamente mais resistentes e rígidos que os de OSB. Com relação à rigidez na direção paralela (MOE paralelo), os painéis foram equivalentes. Conclui-se que a substituição direta do compensado por OSB não é recomendável porque, embora esses painéis possam apresentar propriedades físicas similares como a massa específica aparente, eles também apresentaram propriedades mecânicas diferenciadas, indicando que a substituição desses dois tipos de material deve ser precedida de estudos aprofundados que levem em consideração a magnitude das propriedades requeridas pelos produtos em uso.
\end{abstract}

Palavras-chave: OSB, compensado, resistência, rigidez.

\section{BENDING STRENGTH AND STIFFNESS PROPERTIES OF OSB AND PLYWOOD PANELS}

\begin{abstract}
In a stuffed furniture industry, located in the Piracicaba, SP, Brazil, panel samples (plywood and $O S B$ ) were randomly collected to determine bending strength and stiffness properties (MOR and MOE) in order to compare them and evaluate the possibility of direct substitution of plywood, traditionally used in furniture structural parts, for OSB. The mechanical trials were conducted following NBR 9533 - Brazilian standard, similar to the ASTM D 1037 - American standard. MOR (parallel and perpendicular) and MOE (perpendicular) analyses revealed that the plywood results were significantly higher than the OSB ones. The panels showed to be equivalent with respect to stiffness (MOE parallel). In conclusion, the direct substitution of plywood for OSB is not recommendable, because although they can have similar physical properties (specific gravity) they differed on those important mechanical properties. This indicates that substitutions between those materials should be preceded with more elaborated studies taking into account the magnitude of the properties required by the products in use.
\end{abstract}

Keywords: OSB, plywood, strength, stiffness.

\section{INTRODUÇÃO}

O compensado é um painel formado através da colagem de lâminas de madeira, geralmente em número ímpar de camadas, sobrepostas com a direção das fibras das camadas sucessivas formando ângulos retos entre si (KOLLMANN et al., 1975; TSOUMIS, 1991).

O direcionamento ortogonal de uma lâmina em relação a outra adjacente restringe a movimentação tangencial das camadas e resulta em valores de retratibilidade similares, nas duas direções do plano da cha-

\footnotetext{
${ }^{1}$ Recebido para publicação em 01.4.2003 e aceito para publicação em 10.8.2004.

${ }^{2}$ Departamento de Ciências Florestais da ESALQ/USP. E-mail: < gbortoll@esalq.usp.br/jngarcia@esalq.usp.br>.
} 
pa, conferindo ao compensado excelente estabilidade dimensional. Além disso, o direcionamento cruzado das fibras também proporciona a uniformidade das propriedades mecânicas nas duas direções do plano da chapa. Segundo Tsoumis (1991), essa uniformidade é maior quanto maior é o número de camadas do compensado, em razão da melhor distribuição das tensões que se desenvolvem em face de determinado carregamento.

Outras características importantes dos compensados são a sua capacidade de suportar cargas de impacto que se impõem a certas estruturas e a resistência a rachaduras (WALKER, 1993). De acordo com Tsoumis (1991), é praticamente impossível rachar o compensado, sendo isso também importante no que diz respeito ao uso de pregos e outros conectores.

Existem dois tipos básicos de compensados, sendo um de uso interno, colado principalmente com resinas à base de uréia-formaldeído; e outro de uso externo, em que na maioria das vezes a resina utilizada é de base fenólica (TOMASELLI, 1998). A resina uréica é a mais utilizada na indústria de compensados, de aglomerados e de móveis (NOCK e RICHTER, 1978). Segundo Sellers (2001), na América do Norte se utiliza quase que exclusivamente a resina de fenol-formaldeído para a produção de compensados para usos industrial e estrutural.

Em virtude de possuir diversas características favoráveis, o compensado encontra utilizações das mais variadas, como na construção civil para aplicações estruturais ou não (pisos, forros, paredes, esquadrias, portas, telhados, andaimes, formas de concreto etc.), na construção de barcos e na fabricação de móveis (partes estruturais e decorativas), instrumentos musicais, embalagens industriais, caixas e outros.

O OSB (Oriented Strand Board) é um painel de partículas de madeira orientadas, finas e longas "strands" consolidadas pelo uso de resinas, calor e pressão. De acordo com Sellers (2001), a resina mais utilizada na produção do OSB é a fenólica, entretanto o uso da resina de isocianato é crescente, embora tenha custo superior. Segundo Tsoumis (1991), esse tipo de painel é tipicamente formado por três camadas, sendo nas externas as partículas de madeira orientadas paralelamente na direção de formação do painel; na interna, a orientação é perpendicular. Esse autor afirmou que na essência o OSB é similar ao compensado, no

R. Árvore, Viçosa-MG, v.28, n.4, p.563-570, 2004 que diz respeito à forma de construção, e sua resistência e rigidez à flexão estática e sua estabilidade dimensional são similares.

Os painéis OSB podem ser obtidos a partir de madeiras provenientes de desbastes e de troncos finos e tortuosos, bem como de espécies de menor valor comercial. Competem com os compensados, que requerem toras de alta qualidade para a sua manufatura e, por isso, são de custo relativamente superior (WALKER, 1993).

A principal aplicação do OSB é na indústria da construção civil, pois suas características físicas e mecânicas permitem o uso para fins estruturais. Por esse motivo, esse painel concorre em algumas aplicações com o compensado (TOMASELLI, 1998). O OSB pode expandir-se, consideravelmente, quando estiver exposto a chuvas ou a condições de alta umidade relativa em longos períodos. Devido a isso, deve ser utilizado somente em construções protegidas ou em interiores (JANSSENS, 1998).

O OSB é reconhecido pelos Códigos de Construção do Canadá e dos Estados Unidos como sendo equivalente ao painel de compensado em aplicações estruturais. Três normas de padronização da "Canadian Standards Association" para o OSB são correntemente utilizadas no Canadá, a CSA O437.0 - "OSB and Waferboard", a CSA O325 - “Construction Sheating” e a CSA O452 - “Design Rated OSB”. Nos Estados Unidos, a norma adotada é a US PS 2-92 - "Performance Standard for Structural-Use Wood-Based Panels" (CLOUTIER, 1998; JANSSENS, 1998). Na América Latina, onde não há normas próprias para o OSB, a aceitação dos Códigos de Construção deverá, provavelmente, ocorrer em nível nacional, em conformação com as normas de padronização dos Estados Unidos ou do Canadá (JANSSENS, 1998).

A produção de OSB está basicamente concentrada nos Estados Unidos e no Canadá, sendo esses dois países responsáveis por cerca de $95 \%$ da produção mundial. Os Estados Unidos são responsáveis por mais de $80 \%$ do consumo mundial do produto (TOMASELLI, 1998).

De acordo com estudos desenvolvidos por Howard (2001), uma das maiores mudanças em tecnologia que estavam ocorrendo na indústria à base de madeira era a substituição do compensado pelo OSB no mercado de painéis estruturais dos Estados Unidos. Contu- 
do, dados desse autor evidenciaram que o compensado ainda predominava no ano de 1999 , respondendo por mais de $60 \%$ da produção daquele país.

O OSB não é conhecido no mercado nacional (GOUVEIA et al., 2000). Sua introdução no Brasil ocorreu relativamente recente, sendo originário de importações da Europa. Mesmo antes da sua introdução, as pesquisas desenvolvidas em laboratório sobre esse tipo de painel tiveram início no país, entre as quais, por exemplo, podem ser citadas as publicadas por Gouveia et al. (2000) e Mendes et al. (2000). Embora ainda pouco difundido, o produto já conta com uma fábrica instalada no Brasil, cuja produção, em escala industrial, teve início em 2002.

Previsões feitas por Tuoto e Miyake (2000) indicavam que o OSB deveria ter uma penetração muito rápida no segmento de embalagens e na indústria de construção civil nacionais e que sua produção alcançaria $300.000 \mathrm{~m}^{3}$ no ano de 2004. Segundo o prognóstico desses autores, essa produção corresponderia a 4,6\% do volume total da produção nacional de painéis de madeira, com participações de $8,3 \%$ da chapa dura, de $21,4 \%$ do MDF, de $32,1 \%$ do compensado e de $33,6 \%$ do aglomerado.

O presente trabalho teve por objetivo determinar as propriedades de resistência e de rigidez à flexão estática de painéis OSB e compensados de três diferentes espessuras, com a finalidade de comparálas visando avaliar a possibilidade de substituição direta do compensado, tradicionalmente utilizado nas partes estruturais de móveis estofados, por OSB.

\section{MATERIAL E MÉTODOS}

Em uma indústria de móveis estofados localizada em Piracicaba, SP, na qual se encontrava em estudo a possibilidade de substituição do compensado por OSB nas partes estruturais dos móveis, coletaram-se aleatoriamente corpos-de-prova de um número não determinado de painéis, cortados previamente na própria indústria, para determinação das suas propriedades de resistência (MOR - módulo de resistência) e rigidez (MOE - módulo de elasticidade) à flexão estática. Todos os painéis originais se encontravam estocados na indústria, por mais de 30 dias, em local protegido de variações climáticas acentuadas.

As dimensões dos corpos-de-prova e os ensaios realizados seguiram as prescrições da norma brasileira para compensados NBR 9533 (1986), similar à norma americana ASTM D 1037 (1990). As características e os números de corpos-de-prova, retirados na direção paralela $(/ /)$ e perpendicular $(\perp)$ ao comprimento de cada tipo de painel utilizado no presente estudo, são apresentados no Quadro 1.

Em virtude da indisponibilidade de painéis OSB com espessura de $30 \mathrm{~mm}$ na indústria onde foram coletadas as amostras, optou-se por experimentar a colagem de dois corpos-de-prova de $15 \mathrm{~mm}$ de espessura, efetuada pela própria indústria, e avaliar o seu desempenho em relação ao compensado originalmente manufaturado com $30 \mathrm{~mm}$ de espessura. Nos ensaios de corposde-prova de $30 \mathrm{~mm}$ (compensado e OSB), o vão entre apoios foi igual a 21 vezes a espessura do corpo-deprova, por causa das limitações do dispositivo de ensaio utilizado, portanto diferente do que especifica a norma adotada.

Quadro 1 - Características dos painéis ensaiados e número de corpos-de-prova Table 1 -Characteristics of the tested panels and test specimen number

\begin{tabular}{|c|c|c|c|c|c|c|}
\hline \multirow[t]{2}{*}{ Painel } & \multirow[t]{2}{*}{$\begin{array}{l}\text { Espessura } \\
(\mathrm{mm})\end{array}$} & \multirow[t]{2}{*}{ Tipo de madeira } & \multirow[t]{2}{*}{$\begin{array}{c}\text { Número } \\
\text { de camadas }\end{array}$} & \multirow[t]{2}{*}{ Resina } & \multicolumn{2}{|c|}{$\begin{array}{c}\text { Número de } \\
\text { corpos-de-prova }\end{array}$} \\
\hline & & & & & $/ /$ & $\perp$ \\
\hline$\overline{\text { Compensado }}$ & 15 & Folhosas (Tropicais) & 7 & Uréia-formaldeído & 10 & $\overline{10}$ \\
\hline OSB & 15 & Coníferas(Pinus sp.) & 3 & $\begin{array}{c}\text { Fenol-formaldeído (Externas) } \\
\text { Isocianato (Internas) }\end{array}$ & 09 & 10 \\
\hline Compensado & 18 & Coníferas (Pinus sp.) & 11 & Fenol-formaldeído & 10 & 10 \\
\hline OSB & 18 & Coníferas (Pinus sp.) & 3 & Melamina-uréia-fenol-formaldeído & 10 & 10 \\
\hline Compensado & 30 & Folhosas (Tropicais) & 13 & Uréia-formaldeído & 10 & 10 \\
\hline $\mathrm{OSB}^{1}$ & $(15+15)$ & Coníferas(Pinus sp.) & $(3+3)$ & - & 10 & 10 \\
\hline
\end{tabular}

${ }^{1}$ Dois painéis de $15 \mathrm{~mm}$ colados entre si com resina de uréia-formaldeído pura e catalisador.

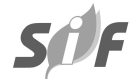

R. Árvore, Viçosa-MG, v.28, n.4, p.563-570, 2004 
Logo após a coleta dos corpos-de-prova, foram realizados os ensaios de flexão estática numa máquina universal de testes com capacidade para aplicação de carga de até seis toneladas, disponível no Laboratório de Engenharia da Madeira da ESALQ/USP. Posteriormente, a partir das extremidades de cada um dos corpos-de-prova ensaiados foram retiradas amostras com dimensões de $75 \times 100 \mathrm{~mm}$, para a determinação da massa específica aparente e do teor de umidade dos painéis.

$\mathrm{Na}$ análise estatística dos dados obtidos dos ensaios realizados, considerou-se cada tipo de painel de determinada espessura como um tratamento. Portanto, foram testados seis tratamentos e os seus efeitos sobre as propriedades avaliadas. Para obtenção desses resultados, realizou-se uma análise de variância e, posteriormente, o teste de Tukey a 5\% de probabilidade. Para a análise da variância, empregou-se o programa estatístico denominado "Statistical Analysis System" (SAS).

\section{RESULTADOS E DISCUSSÃO}

No Quadro 2, apresentam-se os resultados da massa específica aparente, do teor de umidade, do MOR e do MOE dos corpos-de-prova ensaiados à flexão estática. Observa-se nesse quadro que o compensado e o OSB de $15 \mathrm{~mm}$ e o compensado e o OSB de $18 \mathrm{~mm}$ exibiram valores médios de massa específica estatisticamente iguais, tanto nos corpos-de-prova paralelos quanto nos perpendiculares às fibras. Já no compensado $\mathrm{e}$ no OSB de $30 \mathrm{~mm}$ os valores médios da massa específica foram estatisticamente diferentes. O compensado de $30 \mathrm{~mm}$ teve valor inferior ao dos demais.

A massa específica do OSB de $30 \mathrm{~mm}$ foi semelhante à do OSB de $15 \mathrm{~mm}$. Esse resultado é coerente, visto que o OSB de $30 \mathrm{~mm}$ foi obtido pela colagem de dois painéis OSB de $15 \mathrm{~mm}$.

Os valores médios do teor de umidade dos compensados foram compatíveis aos disponíveis na literatura. Kollmann, citado por Kollmann et al. (1975), com base em ensaios com compensados produzidos por diferentes fabricantes, informaram que o teor de umidade desses painéis, após serem estocados em ambiente normalizado, variou entre 7,3 e 12,7\%. Isso se deve às altas temperaturas empregadas na secagem de lâminas e na prensagem a quente do compensado, o que reduz sua capacidade de sorção (KOLLMANN e SCHNEIDER, citados por KOLLMANN et al., 1975), e aos adesivos sintéticos termorrígidos, que, quando curados, não são higroscópicos (KOLLMANN et al., 1975). Acredita-se que essas mesmas afirmações feitas para lâminas também sejam válidas para partículas de madeira.

Quadro 2 - Valores médios da massa específica aparente, teor de umidade, MOR e MOE obtidos de corpos-de-prova de compensado e OSB ensaiados à flexão estática na direção paralela e perpendicular às fibras

Table 2 -Specific gravity, moisture content, MOR and MOE mean values obtained from plywood and OSB test specimens in the static bending-perpendicular-to-grain and static bending-parallel-to-grain trials

\begin{tabular}{|c|c|c|c|c|c|c|c|c|}
\hline \multirow[b]{3}{*}{ Tratamentos } & \multicolumn{8}{|c|}{ Flexão Estática } \\
\hline & \multicolumn{4}{|c|}{ Paralela às fibras } & \multicolumn{4}{|c|}{ Perpendicular às fibras } \\
\hline & $\begin{array}{c}\text { Massa específica } \\
\text { aparente } \\
\left(\mathrm{g} / \mathrm{cm}^{3}\right)\end{array}$ & $\begin{array}{c}\text { Teor de } \\
\text { umidade }(\%)\end{array}$ & $\begin{array}{c}\text { MOR } \\
(\mathrm{MPa})\end{array}$ & $\begin{array}{l}\mathrm{MOE} \\
(\mathrm{MPa})\end{array}$ & $\begin{array}{c}\text { Massa específica } \\
\text { aparente } \\
\left(\mathrm{g} / \mathrm{cm}^{3}\right)\end{array}$ & $\begin{array}{c}\text { Teor de } \\
\text { umidade } \\
(\%)\end{array}$ & $\begin{array}{c}\text { MOR } \\
(\mathrm{MPa})\end{array}$ & $\begin{array}{l}\mathrm{MOE} \\
(\mathrm{MPa})\end{array}$ \\
\hline $\begin{array}{l}\text { Compensado } \\
(15 \mathrm{~mm})\end{array}$ & $0,55 \mathrm{~B}$ & 12,1 & $34 \mathrm{AB}$ & $4474 \mathrm{~B}$ & $0,57 \mathrm{~B}$ & 12,1 & $32 \mathrm{~A}$ & $3606 \mathrm{~B}$ \\
\hline $\begin{array}{l}\text { OSB } \\
(15 \mathrm{~mm})\end{array}$ & $0,56 \mathrm{~B}$ & 9,0 & $25 \mathrm{C}$ & $3987 \mathrm{BC}$ & $0,57 \mathrm{~B}$ & 8,9 & $16 \mathrm{~B}$ & $1756 \mathrm{D}$ \\
\hline $\begin{array}{l}\text { Compensado } \\
(18 \mathrm{~mm})\end{array}$ & $0,63 \mathrm{~A}$ & 10,9 & $40 \mathrm{~A}$ & $5932 \mathrm{~A}$ & $0,60 \mathrm{~A}$ & 10,5 & $36 \mathrm{~A}$ & $3966 \mathrm{AB}$ \\
\hline $\begin{array}{l}\text { OSB } \\
(18 \mathrm{~mm})\end{array}$ & $0,62 \mathrm{~A}$ & 8,7 & $32 \mathrm{~B}$ & $5671 \mathrm{~A}$ & $0,63 \mathrm{~A}$ & 8,5 & $19 \mathrm{~B}$ & $2774 \mathrm{C}$ \\
\hline $\begin{array}{l}\text { Compensado } \\
(30 \mathrm{~mm})\end{array}$ & $0,48 \mathrm{C}$ & 12,7 & $35 \mathrm{AB}$ & $4665 \mathrm{~B}$ & $0,49 \mathrm{C}$ & 12,6 & $35 \mathrm{~A}$ & $4424 \mathrm{~A}$ \\
\hline $\begin{array}{l}\text { OSB } \\
(30 \mathrm{~mm})\end{array}$ & $0,57 \mathrm{~B}$ & 9,4 & $18 \mathrm{D}$ & $3216 \mathrm{C}$ & $0,56 \mathrm{~B}$ & 9,0 & $15 \mathrm{~B}$ & $2228 \mathrm{D}$ \\
\hline
\end{tabular}

*Médias seguidas por uma mesma letra na vertical não diferem estatisticamente, pelo teste de Tukey a 5\% de probabilidade. 
Por causa das características do processo de produção do compensado, bem como das características específicas dos dois tipos de adesivos e das formulações empregadas em cada caso, os compensados colados com resina uréica são mais higroscópicos que os fenólicos. Por isso, o teor de umidade do compensado de $18 \mathrm{~mm}$ (fenólico) foi inferior ao dos demais compensados uréicos.

No caso dos painéis OSB, os teores de umidade foram inferiores aos dos compensados. Isso se justifica, pois no processo de produção do OSB a colagem requer partículas com teores de umidade variáveis entre 2 e $6 \%$, dependendo do tipo de adesivo empregado e das temperaturas de prensagem do colchão, que variam entre $200 \mathrm{e} 220^{\circ} \mathrm{C}$ (CLOUTIER, 1998). Em razão da exposição das partículas a altas temperaturas, tanto na secagem quanto na prensagem, bem como das características dos adesivos sintéticos e dos aditivos utilizados na produção do OSB, esse painel alcança teores de umidade de equilíbrio inferiores ao do compensado, quando ambos os tipos de painéis são expostos em ambiente com umidade relativa e temperatura equivalente.

Com base no que dispõe a literatura, bem como na massa específica, tomando-se como exemplo o compensado e o OSB de $15 \mathrm{~mm}$ e o compensado e o OSB de $18 \mathrm{~mm}$, cujos valores médios foram estatisticamente iguais, poderiam ser esperados valores de resistência e rigidez equivalentes, porém isso não se verificou inteiramente, por que tal equivalência somente ocorreu no MOE paralelo.

Como pode ser observado no Quadro 2, houve diferenças significativas no MOR paralelo entre o compensado e OSB de mesma espessura. Os valores médios do MOR paralelo nos compensados de 15, 18 e $30 \mathrm{~mm}$ foram 26, 20 e $49 \%$ superiores aos valores obtidos nos painéis de OSB.

O valor médio do MOR paralelo obtido no OSB de $30 \mathrm{~mm}$ foi inferior a todos os demais, indicando claramente que a colagem entre as duas peças de OSB de $15 \mathrm{~mm}$ foi ineficiente no sentido de se obter um valor de resistência aceitável. Inclusive, durante o ensaio de flexão, alguns corpos-de-prova se descolaram na região da junção colada, provavelmente pelo efeito do cisalhamento, e foram excluídos da análise estatística efetuada. Portanto, nas condições utilizadas, pode-se afirmar que não é aconselhável a colagem de duas peças de OSB de $15 \mathrm{~mm}$, pois, nessas mesmas condições, a resistência à flexão estática, especialmente na direção paralela, foi inferior àquela obtida a partir de uma única peça de $15 \mathrm{~mm}$. Também, é possível supor que o desempenho de duas peças de OSB de $15 \mathrm{~mm}$ coladas entre si não seja o mesmo que o de uma única peça de OSB manufaturada originalmente com espessura de $30 \mathrm{~mm}$, da qual se pode esperar melhor desempenho, por causa de uma presumida ausência de ocorrência de cisalhamento, de maneira acentua$\mathrm{da}$, como se verificou nas peças coladas.

Entre os compensados de todas as espessuras houve equivalência estatística nos valores médios de MOR paralelo. Isso não ocorreu entre os painéis de OSB, cujos valores médios diferiram estatisticamente entre si. No entanto, houve equivalência estatística dos valores médios do MOR paralelo entre o OSB de $18 \mathrm{~mm}$ e os compensados de 15 e $30 \mathrm{~mm}$, demonstrando que nessa propriedade o OSB revelou bom desempenho, embora inferior ao do compensado de $18 \mathrm{~mm}$.

No MOR perpendicular, também houve diferenças significativas entre o compensado e OSB de espessuras semelhantes, que na sua maioria foram ainda mais acentuadas que as verificadas no MOR paralelo. Os valores médios do MOR perpendicular dos compensados de 15,18 e $30 \mathrm{~mm}$ foram 50,53 e $43 \%$ superiores aos valores obtidos nos painéis OSB. Certamente, essas diferenças ocorrem por causa das características de cada tipo de painel, especialmente no que diz respeito ao número de camadas, que é maior no compensado (Quadro 1), proporcionando-lhe resistência mais elevada na direção perpendicular, bem como uma resistência mais uniforme entre as direções paralela e perpendicular.

Os valores resultantes da relação MOR paralelo e MOR perpendicular foram de 1,06; 1,11; e 1,00 nos compensados e de 1,$56 ; 1,68$; e 1,2 nos painéis OSB de 15,18 e $30 \mathrm{~mm}$, respectivamente. Observou-se que a referida relação foi mais favorável no compensado, ou seja, a diferença entre a resistência à flexão estática paralela e perpendicular foi notavelmente menor nos compensados de todas as espessuras ou praticamente desprezível, conforme ilustra a Figura 1. O resultado prático dessas constatações é que peças de OSB, cujos comprimentos forem perpendiculares à orientação das partículas das camadas externas, encontrarão aplicações mais restritas em virtude da redução da sua resistência, e isso pode interferir no percentual de aproveitamento do painel.

R. Árvore, Viçosa-MG, v.28, n.4, p.563-570, 2004 


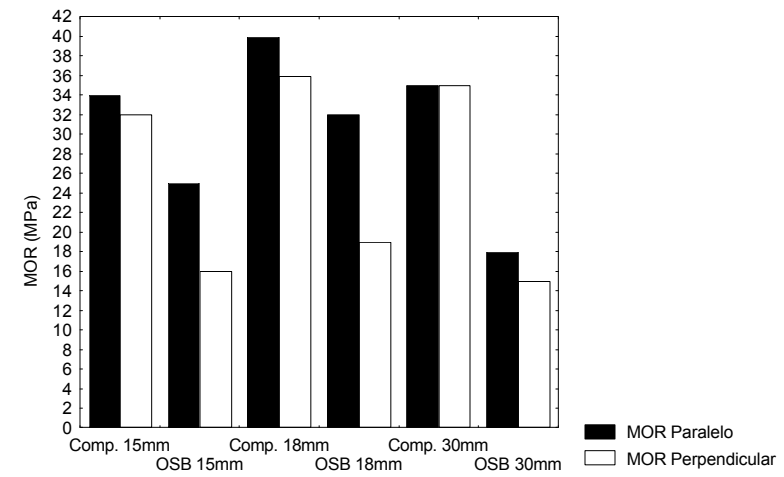

Figura 1 - Valores médios comparativos de MOR paralelo e MOR perpendiculares aos painéis compensados e OSB.

Figure 1-Comparative mean values of MOR-perpendicularto-grain and MOR-parallel-to-grain from plywood and $O S B$ panels.

Entre os compensados de todas as espessuras houve equivalência estatística dos valores médios de MOR perpendicular, tendo ocorrido o mesmo entre os painéis OSB.

Os valores médios de MOE paralelo se equivaleram estatisticamente no compensado e OSB de $15 \mathrm{~mm}$, verificando-se o mesmo no compensado e OSB de $18 \mathrm{~mm}$. Esses resultados indicam que, na propriedade em referência, há similaridade entre compensado e OSB, corroborando o disposto na literatura.

No caso do compensado e OSB de $30 \mathrm{~mm}$, os valores médios foram significativamente diferentes, sendo inferiores no OSB de $30 \mathrm{~m}$ que, por sua vez, foram equivalente ao valor médio do MOE paralelo do OSB de $15 \mathrm{~mm}$.

Os valores médios do MOE paralelo ainda se equivaleram estatisticamente nos compensados de 15 e $30 \mathrm{~mm}$ e OSB de $15 \mathrm{~mm}$.

No MOE perpendicular houve diferenças significativas entre o compensado e OSB de mesmas espessuras. Os valores médios do MOE perpendicular nos compensados de 15, 18 e $30 \mathrm{~mm}$ foram 51, 30 e $50 \%$ superiores aos obtidos nos painéis OSB.

Conforme ilustra a Figura 2, a análise da relação MOE paralelo e MOE perpendicular no compensado e OSB de mesma espessura foi favorável ao compensado, de maneira ainda mais acentuada do que evidenciou a análise da relação MOR paralelo e MOR

R. Árvore, Viçosa-MG, v.28, n.4, p.563-570, 2004

R. Arvore, Viçosa-MG, v.28, n.4, p.563-570, 2004 perpendicular. Conforme comentado anteriormente, esse fato está relacionado ao número de camadas que compõem os painéis.

De acordo com Cloutier (1998), a norma CSA 0437.0 (1993) estabelece duas classes de painéis OSB, sendo O-1 e O-2. Para o OSB ser enquadrado na classe O-1, os valores mínimos de resistência e rigidez são os seguintes: MOR paralelo e perpendicular de 23,4 e 9,6 MPa, respectivamente; e MOE paralelo e perpendicular de 4500 e $1300 \mathrm{MPa}$, respectivamente. Para o OSB ser enquadrado na classe O-2, os valores mínimos de resistência e rigidez exigidos são os seguintes: MOR paralelo e perpendicular de 29,0 e 12,4 MPa, respectivamente; e MOE paralelo e perpendicular de 5.500 e $1.500 \mathrm{MPa}$.

Os valores do Quadro 2, quando confrontados com aqueles prescritos pela norma CSA 0437.0 (1993), indicaram que o OSB de $18 \mathrm{~mm}$ atende inteiramente aos requisitos da classe $\mathrm{O}-2$, demonstrando o bom desempenho desse painel, como comentado anteriormente. O OSB de $15 \mathrm{~mm}$ não atende inteiramente aos requisitos da classe $\mathrm{O}-1$, pois o valor médio do $\mathrm{MOE}$ paralelo é inferior ao mínimo exigido pela norma em referência. $\mathrm{O} O S B$ de $30 \mathrm{~mm}$ definitivamente não atende sequer aos requisitos da classe $\mathrm{O}-1$, pois os valores médios do MOR paralelo, do MOE paralelo e do MOE perpendicular foram bem inferiores ao mínimo exigido pela norma. Essas constatações reforçam, ainda mais, a inadequação da colagem de duas peças de $15 \mathrm{~mm}$.

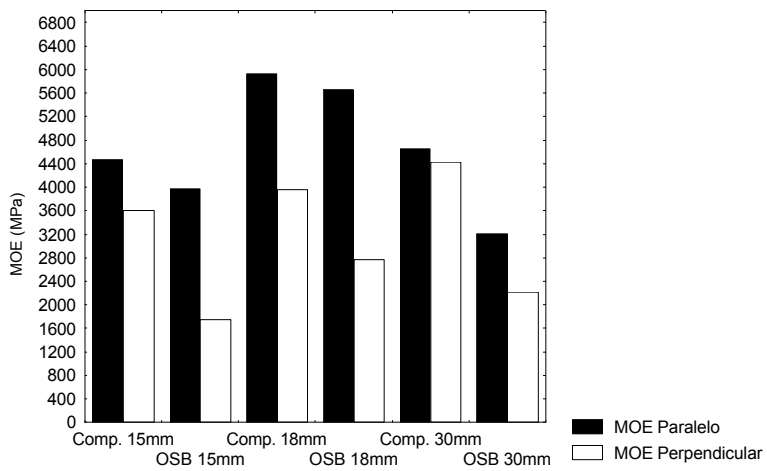

Figura 2 - Valores médios comparativos de MOE paralelo e MOE perpendicular aos painéis compensados e OSB.

Figure 2-Comparative mean values of $M O E$-perpendicularto-grain and MOE-parallel-to-grain to plywood and OSB panels. 
Utilizando os requisitos da norma CSA 0437.0 (1993) somente como referência, pode-se observar que o compensado de $15 \mathrm{~mm}$ não atenderia inteiramente aos requisitos da classe $\mathrm{O}-1$, especialmente por que o valor médio do MOE paralelo foi pouco inferior ao mínimo especificado, embora tenha apresentado valores médios de MOR paralelo, MOR perpendicular e MOE perpendicular bastante superiores aos exigidos. O compensado de $18 \mathrm{~mm}$ atenderia inteiramente aos requisitos da classe $\mathrm{O}-2$, com boa margem de segurança. $\mathrm{O}$ compensado de $30 \mathrm{~mm}$ somente poderia ser admitido na classe $\mathrm{O}-1$, em razão de o valor médio do MOE paralelo ter sido inferior ao mínimo exigido para enquadrá-lo na classe $\mathrm{O}-2$, embora os demais valores permitissem tal enquadramento com excelente margem de segurança.

Segundo Pereyra (1994), a norma DIN 68705 (1981) exige os seguintes valores mínimos de resistência para compensados de construção: MOR paralelo e perpendicular de 40 e $15 \mathrm{MPa}$, respectivamente. Cabe comentar que o fato de essa norma não considerar valores de rigidez se configura como certa limitação, uma vez que o MOE é uma importante propriedade. Entretanto, deve ser levado em conta que MOR e MOE se correlacionam bem.

A partir do conjunto dos resultados obtidos em cada ensaio individual, cujos valores médios se encontram no Quadro 2, correlações foram estabelecidas entre MOR e MOE paralelo e entre MOR e MOE perpendicular, nos dois tipos de painéis. Nos compensados, os resultados foram 0,67 e 0,60 , respectivamente. Nos painéis OSB, os resultados foram 0,92 e 0,52 , respectivamente. Essas correlações foram significativas a $5 \%$ de probabilidade.

Confrontando os valores do Quadro 2 com os requisitos da norma DIN 68705 (1981), observou-se que somente o compensado de $18 \mathrm{~mm}$ atendeu aos valores mínimos exigidos, demonstrando o bom desempenho desse painel, que se destacou entre todos os demais avaliados. Os outros compensados (15 e $30 \mathrm{~mm}$ ) não se enquadraram nos requisitos da norma, especialmente por causa dos valores de MOR paralelo, que eram inferiores ao mínimo exigido, embora os valores de MOR perpendicular sejam maiores que o dobro do mínimo especificado.

Ao considerar o que dispõe a norma DIN 68705 (1981), pode-se observar que todos os painéis OSB avaliados no presente estudo não poderiam atender ao valor mínimo exigido para o MOR paralelo.

\section{CONCLUSÕES}

Os compensados e OSB de 15 e $18 \mathrm{~mm}$ não apresentaram diferença significativa com relação à massa específica. Nessa propriedade, o compensado de 30 $\mathrm{mm}$ apresentou valor inferior a todos os demais painéis.

Houve diferença significativa entre os compensados e OSB de mesma espessura no MOR paralelo, MOR perpendicular e MOE perpendicular, cujos valores foram superiores nos compensados em todos os casos, contrariando o que dispõe a literatura.

Os valores do MOE paralelo dos compensados e OSB de 15 e $18 \mathrm{~mm}$ foram similares, corroborando o que dispõe a literatura. No caso dos painéis de 30 $\mathrm{mm}$, o compensado apresentou valor superior nessa propriedade.

Os compensados de todas as espessuras apresentaram maior uniformidade em resistência e rigidez entre as duas direções do plano dos painéis, em relação ao OSB.

Dentre todos os painéis estudados, o OSB de 30 $\mathrm{mm}$ foi o que apresentou o desempenho menos satisfatório, indicando que a colagem de duas peças de OSB de 15 mm não é aconselhável.

A substituição direta do compensado por OSB não é recomendável porque, embora esses painéis possam apresentar propriedades físicas similares como a massa específica aparente, eles exibiram importantes propriedades mecânicas diferenciadas, indicando que a substituição entre esses dois tipos de material deve ser precedida de estudos aprofundados que levem em consideração a magnitude das propriedades requeridas pelos produtos em uso.

\section{REFERÊNCIAS BIBLIOGRÁFICAS}

\section{ASSOCIAÇÃO BRASILEIRA DE NORMAS TÉCNICAS - ABNT. NBR-9533. Chapas de madeira compensada - Método de ensaio: determinação da resistência à flexão estática. Rio de Janeiro: 1986.}

\begin{abstract}
AMERICAN SOCIETY FOR TESTING AND MATERIALS - ASTM. Standard test methods of evaluating the properties of wood-base fiber and particle panel
\end{abstract} materials. ASTM D 1037-89. Philadelphia: 1990. 
CANADIAN STANDARDS ASSOCIATION - CSA. OSB and Waferboard. CSA 0437.0-93.

Ontario: 1993.

CLOUTIER, A. Oriented strandboard (OSB): raw material, manufacturing process, properties and use. In: SEMINÁRIO INTERNACIONAL SOBRE PRODUTOS SÓLIDOS DE MADEIRA DE ALTA TECNOLOGIA, 1., 1998, Belo Horizonte-MG. Anais...Viçosa-MG: SIF/UFV/DEF, p. 173-185, 1998.

DEUTSCHES INSTITUT FÜR NORMUNG - DIN. Plywood: building-veneer plywood. DIN 68705-3. Berlin: 1981.

GOUVEIA, F.N.; SANTANA, MA.E.; SOUZA, M.R. Utilização da madeira de Eucalyptus grandis e Eucalyptus urophylla na fabricação de chapas de partículas orientadas (OSB) e não-orientadas. Revista Árvore, v.24, n.1, p. 7-12, 2000.

HOWARD, J.L. U.S. timber production, trade, consumption and price statistics 1965 to 1999. USDA, n ${ }^{\circ}$ FPL-RP-595, 2001.

JANSSENS, D.P. The increasing recognition of oriented strand board (OSB) as a preferred structural panel. In: SEMINÁRIO INTERNACIONAL SOBRE PRODUTOS SÓLIDOS DE MADEIRA DE ALTA TECNOLOGIA, 1., 1998, Belo Horizonte-MG. Anais...Viçosa-MG: SIF/ UFV/DEF, 1998. p. 169-172.

KOLLMANN, F.F.P.; KUENZI, E.W.; STAMM, A.J. Principles of wood science and technology. Berlin: Springer-Verlag, 1975. v.2, p. 154-283, 1975.
MENDES, L.M. et al. Avaliação do sistema de orientação de partículas na produção de painéis OSB (Oriented Strand Board). Revista Cerne, v.6, n.1, p. 1-8, 2000.

NOCK, H.P.; RICHTER, H.G. Adesão e adesivos. Curitiba: UFPR/SCA/CPGEF, 1978. 94p.

PEREYRA, O. Avaliação da madeira de Eucalyptus dunnii (Maid) na manufatura de painéis compensados . 1994. $87 \mathrm{f}$.

Dissertação (Mestrado em Ciência e Tecnologia da Madeira) - Escola Superior de Agricultura "Luiz de Queiroz", Piracicaba, 1994.

SELLERS, T. Wood adhesive: innovations and applications in North America. Forest

Products Journal, v. 51, n. 6, june 2001, p. 12-22.

TOMASELLI, I. A indústria de painéis no Brasil e no mundo: tendências de mudanças do perfil de produção e usos. In: SEMINÁRIO

INTERNACIONAL SOBRE PRODUTOS SÓLIDOS DE MADEIRA DE ALTA TECNOLOGIA, 1., 1998, Belo Horizonte-MG. Anais...Viçosa-MG: SIF/ UFV/DEF, 1998. p. 55-64.

TSOUMIS, G. Science and technology of wood: structure, properties, utilization. New York: Chapman \& Hall, 1991. p. 309-339.

TUOTO, M.; MIYAKE, N. A indústria de painéis de madeira supera as expectativas.

Informativo STCP, n. 4, p. 20-22, 2000.

WALKER, J.C.F. Primary wood processing: principles and practice. London: Chapman \& Hall, 1993. p. 377-416. 